\title{
COLORECTAL SURGERY
}

\section{Effectiveness of a care bundle to reduce surgical site infections in patients having open colorectal surgery}

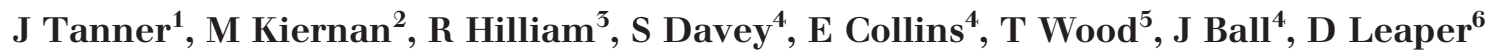 \\ ${ }^{1}$ University of Nottingham, UK \\ ${ }^{2}$ University of West London, UK \\ ${ }^{3}$ Open University, UK \\ ${ }^{4}$ University Hospitals of Leicester NHS Trust, UK \\ ${ }^{5}$ University of Leicester, UK \\ ${ }^{6}$ University of Huddersfield, UK \\ ABSTRACT \\ INTRODUCTION In 2010 a care bundle was introduced by the Department of Health (DH) to reduce surgical site infections \\ (SSIs) in England. To date, use of the care bundle has not been evaluated despite incorporating interventions with resource \\ implications. The aim of this study was to evaluate the DH SSI care bundle in open colorectal surgery. \\ METHODS A prospective cohort design was used at two teaching hospitals in England. The baseline group consisted of 127 \\ consecutive patients having colorectal surgery during a 6-month period while the intervention group comprised 166 patients in \\ the subsequent 6 months. SSI and care bundle compliance data were collected using dedicated surveillance staff. \\ RESULTS Just under a quarter (24\%) of the patients in the baseline group developed a SSI compared with just over a quarter \\ $(28 \%)$ in the care bundle group $(p>0.05)$. However, compliance rates with individual interventions, both before and after the \\ implementation of the bundle, were similar. Interestingly, in only 19\% of cases was there compliance with the total care bun- \\ dle. The single intervention that showed an associated reduction in SSI was preoperative warming $(p=0.032)$. \\ CONCLUSIONS The DH care bundle did not reduce SSIs after open colorectal surgery. Despite this, it is not possible to state \\ that the bundle is ineffective as compliance rates before and after bundle implementation were similar. All studies evaluating \\ the effectiveness of care bundles must include data for compliance with interventions both before and after implementation of \\ the care bundle; poor compliance may be one of the reasons for the lower than expected reduction of SSIs.
}

\author{
KEYWORDS \\ Colorectal surgery - Surgical site infection - Care bundle - Compliance \\ Accepted 28 September 2015 \\ CORRESPONDENCE TO \\ Judith Tanner, E: judith.tanner@nottingham.ac.uk
}

The potential for the use of care bundles was first proposed by the Institute for Healthcare Improvement in 2001 to improve outcomes in critical care. ${ }^{1}$ With the bundle approach, a minimum of three robust, evidence-based interventions are implemented collectively and consistently so that there is a possible summation of their effects to reduce complications or adverse events. The first care bundles were directed at reducing ventilator associated pneumonia (VAP) and central line associated bloodstream infections (CLABSIs); these demonstrated considerable success. ${ }^{2,3}$ Following on from these successes, the focus and range of bundles was extended to address other clinical challenges including surgical site infections (SSIs). SSIs have the potential to be reduced by a bundle approach because there are many associated risk factors to target and there are a considerable number of robust, evidence-based interventions.

Care bundles to reduce SSIs have been introduced at national levels, such as the Surgical Care Improvement Project (SCIP) in the US and the Department of Health's High Impact Intervention (DH HII) in England. ${ }^{4,5}$ SCIP was launched in 2006 with core interventions of antibiotic prophylaxis and appropriate preoperative hair removal as well as additional interventions to maintain normothermia in colorectal patients and glycaemic control in cardiac patients. The project was organised by a US surgical care partnership and $95 \%$ compliance with interventions is required to receive full reimbursement from Medicaid. ${ }^{6}$ There have been mixed reports of its effectiveness. ${ }^{7,8}$ 
The English DH HII bundle was introduced in 2010. It was based on the recommendations of a systematic review of interventions to reduce SSIs, expert advice, and other national and international infection prevention and control guidance by the National Institute for Health and Clinical Excellence. ${ }^{9}$ It includes interventions to address the three phases: pre, intra and postoperative care. A systematic review and meta-analysis has shown that care bundles can reduce the risk of SSI after colorectal surgery ${ }^{10}$ but to date, no evaluation of the DH HII bundle for the prevention of SSI after colorectal surgery has been published. The effectiveness of this latter care bundle needs evaluation as many of the component interventions have significant financial implications, and may require additional resources and staffing to implement.

The aim of this study was to address the following questions:

$>$ Does implementation of the DH HII bundle reduce SSIs following open colorectal surgery?

$>$ Is compliance with the entire bundle associated with a lower rate of SSI than incomplete compliance?

\section{Methods}

The DH HII care bundle was evaluated using a prospective cohort design at two large teaching hospitals in England. SSI data from the baseline cohort were collected by a dedicated surveillance team over a six-month period. The bundle was then introduced and implemented, and intervention cohort data were collected during the subsequent six months. Cohort compliance with care bundle interventions was measured by a project assistant and SSIs were measured by the surveillance team.

The relevant hospital ethics committee stated that approval was not required as SSI data were already being routinely collected. However, permission to analyse anonymised SSI data was required and was given.

Data were collected for all consecutive adult patients having the following open colorectal procedures: left or right hemicolectomy, extended right hemicolectomy, anterior resection, abdominoperineal resection, total colectomy, sigmoid colectomy, Hartmann's procedure or subtotal colectomy. Laparoscopic procedures were excluded.

\section{Care bundle interventions}

The DH HII care bundle is split into three elements: the preoperative, intraoperative and postoperative phases. The nine interventions of the bundle, incorporating all three phases, are:

1. screening and decolonisation for methicillin resistant Staphylococcus aureus (MRSA)

2. preoperative showering with soap

3. appropriate hair removal using single use clipper heads if hair removal is needed

4. intravenous antibiotic prophylaxis, administered up to 60 minutes before incision, at the correct dose

5. skin preparation with $2 \%$ chlorhexidine in $70 \%$ alcohol
6. maintenance of normothermia in the perioperative period (In this study, this involved use of forced air warming $[\mathrm{FAW}]$ blankets in the operating theatre. FAW blankets were also given to patients for one hour before transfer to the operating room.)

7. use of antiseptic impregnated incise drapes

8. use of supplemental oxygen in the early postoperative phase

9. glucose control for diabetic patients throughout the three phases

The SSI rates and compliance data collected during the study were posted in key areas such as scrub rooms, and they were updated monthly.

\section{SSI data collection}

Continuous SSI surveillance following colorectal surgery was well established in the two hospitals contributing to the study, having commenced two years before implementation of the DH HII bundle and continuing after the bundle evaluation was completed. All patients undergoing open colorectal surgery were included in the surveillance programme. Surveillance was undertaken by dedicated staff, based in infection prevention and control teams, who were already collecting SSI data and patient demographics through direct patient contact, patient notes and hospital electronic information systems. After discharge, follow-up data were collected via telephone calls with patients on the 15th and 30th postoperative day. The Health Protection Agency (now part of Public Health England) definition of a SSI was used, ${ }^{11}$ which is a modified version of the US Centers for Disease Control definition. ${ }^{12}$ Data entry and quality control checks were performed by the lead surveillance nurse.

\section{Compliance data collection}

A project assistant was employed to monitor compliance with bundle interventions. Compliance data were collected for each individual intervention and also for the bundle in totality. Direct observation of clinical practice was also undertaken by the project assistant to confirm the validity of documented data.

\section{Statistical analysis}

Compliance data, SSI data and patient characteristics for each patient were entered into Excel $^{\circledR}$ (Microsoft, Redmond, WA, US). Data were anonymised and identifying fields removed prior to analysis. The characteristics of the baseline and care bundle groups were compared, and SSI rates in the six-month period immediately before the implementation of the bundle were compared with SSI rates in the six months after bundle implementation using chi-squared analysis. Similarly, individual interventions that may have had an association with a subsequent SSI were determined. In order to measure compliance with the total care bundle, each intervention was given a score of one point. Compliance with all nine interventions therefore gave a maximum score of nine points. 


\section{Results}

The baseline group comprised 127 patients undergoing open colorectal surgery during the six-month period before the DH HII care bundle was implemented while the intervention group consisted of 166 patients undergoing surgery during the 6 months after implementation. An additional 11 patients were lost to follow-up and excluded from the surveillance programme during this time.

In the baseline group, 31 patients $(24 \%)$ developed a SSI compared with $46(28 \%)$ in the care bundle group. This was not statistically significant $(p=0.525)$. In terms of just superficial incisional SSIs, these were found in 11 patients (9\%) in the baseline group and 28 patients $(17 \%)$ in the care bundle group. This finding was statistically significant $(p=0.040)$. With regard to deep incisional or organ space SSIs, 20 patients (15\%) in the baseline group developed these compared with 18 patients $(11 \%)$ in the care bundle group, which was not statistically significant $(p=0.216)$. The characteristics of the two groups were comparable (Table 1).

Almost all of the interventions listed in the DH HII care bundle (apart from use of $2 \%$ chlorhexidine gluconate in $70 \%$ alcohol and preoperative warming) had already been introduced at the hospitals prior to the implementation of the bundle. With the exception of maintenance of intraoperative normothermia, baseline compliance with preexisting individual interventions was already high and did not increase significantly following formal implementation of the bundle (Table 2). Conversely, compliance with maintenance of normothermia remained low following bundle implementation, with a small increase from $23 \%$ to $35 \%$.

\begin{tabular}{|c|c|c|c|}
\hline & $\begin{array}{l}\text { Baseline } \\
\text { group } \\
(n=127)\end{array}$ & $\begin{array}{l}\text { Care bundle } \\
\text { group } \\
(n=166)\end{array}$ & $p$-value \\
\hline Mean age in years & $\begin{array}{l}63.5 \\
\text { (SD: 16.4) }\end{array}$ & $\begin{array}{l}64.1 \\
\text { (SD: 15.3) }\end{array}$ & 0.755 \\
\hline $\begin{array}{l}\text { Sex } \\
\text { Male } \\
\text { Female }\end{array}$ & $\begin{array}{l}63(50 \%) \\
63(50 \%)\end{array}$ & $\begin{array}{l}83(50 \%) \\
83(50 \%)\end{array}$ & 0.947 \\
\hline Smoker & $21(17 \%)$ & 20 (12\%) & 0.273 \\
\hline Diabetic & $14(11 \%)$ & $28(16 \%)$ & 0.157 \\
\hline $\begin{array}{l}\text { Mean BMI in } \\
\mathrm{kg} / \mathrm{m}^{2}\end{array}$ & $\begin{array}{l}26.38 \\
\text { (SD: } 4.98)\end{array}$ & $\begin{array}{l}26.58 \\
\text { (SD: } 5.34)\end{array}$ & 0.740 \\
\hline Elective admission & 88 (69\%) & $103(62 \%)$ & 0.197 \\
\hline $\begin{array}{l}\text { NNIS risk score* } \\
0 \\
1 \\
2 \\
3\end{array}$ & $\begin{array}{l}40(31 \%) \\
49(39 \%) \\
32(25 \%) \\
6(5 \%)\end{array}$ & $\begin{array}{l}38(23 \%) \\
73(44 \%) \\
50(30 \%) \\
5(3 \%)\end{array}$ & 0.297 \\
\hline \multicolumn{4}{|c|}{$\begin{array}{l}\text { SD = standard deviation; BMI = body mass index; } \\
\text { NNIS = National Nosocomial Infections Surveillance } \\
\text { *calculated from ASA grade, wound class and duration of surgery }\end{array}$} \\
\hline
\end{tabular}

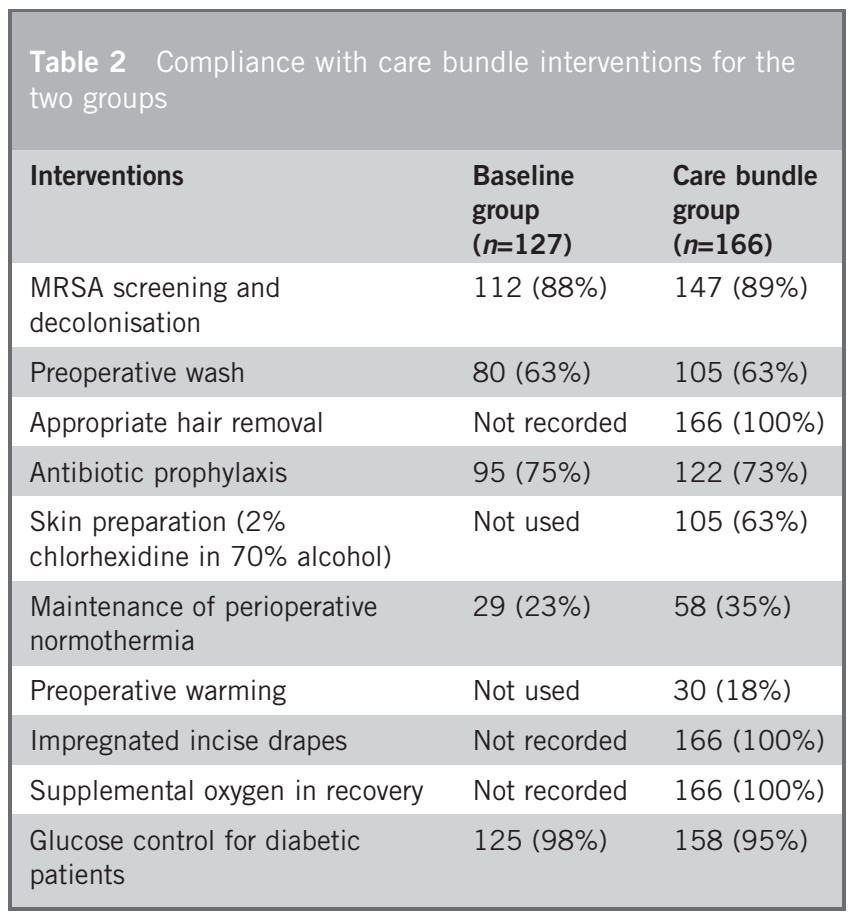

The uptake of the new interventions was mixed. For example, compliance of use of $2 \%$ chlorhexidine gluconate skin preparation reached $63 \%$ while only $18 \%$ of patients received preoperative warming in the bundle period. However, preoperative warming proved to be challenging to implement as the logistics were more complex. (Staff required training in numerous wards and warming devices needed hospital testing and approval.)

After the introduction of the care bundle, all patients received at least four of the nine interventions in the bundle (Figure 1). Nevertheless, only 19\% (32/166) of patients received the bundle in its entirety.

Figure 1 also shows the SSI rates among patients who received increasing numbers of interventions. There were insufficient data to determine whether compliance with

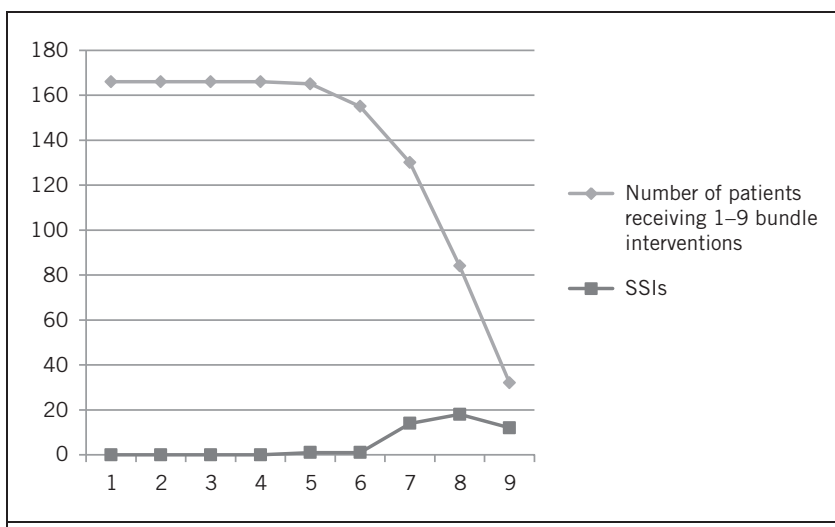

Figure 1 Compliance with bundle interventions and surgical site infections 


\begin{tabular}{|llll|}
\hline $\begin{array}{l}\text { Table } 3 \\
\text { (SSI) }\end{array}$ & Preoperative warming and surgical site infection & \\
& SSI & No SSI & Total \\
\hline Preoperative warming & 3 & 27 & 30 \\
\hline No preoperative warming & 74 & 189 & 263 \\
\hline Total & 77 & 216 & 293 \\
\hline
\end{tabular}

implementation of the whole bundle was associated with a greater reduction in SSIs than receipt of only some elements of the bundle.

Chi-squared analysis demonstrated no relationship between the following individual interventions and SSI: MRSA screening and decolonisation, preoperative wash, antibiotic prophylaxis, maintenance of normothermia, impregnated incise drapes, supplemental oxygen, glucose control for diabetics or $2 \%$ chlorhexidine in alcohol. However, preoperative warming was associated with a significantly lower SSI rate (10\% vs $28 \%, p=0.032$ ) (Table 3).

\section{Discussion}

It would be tempting to draw the conclusion from these results that the DH HII care bundle was not effective among this cohort of patients having colorectal surgery. Nevertheless, compliance with most interventions remained similar after the implementation of the bundle and only $19 \%$ of patients actually received the entire bundle. It is therefore not possible to comment on the effectiveness of the bundle in totality. However, this study identifies the importance of compliance rates in care bundle studies, data that are frequently not provided.

As care bundles comprise a group of interventions that constitute best practice, it would be expected that many components of a bundle (such as appropriate and timely antibiotic prophylaxis or maintaining normothermia) would already be routine practice in perioperative care. Consequently, all studies evaluating care bundles, especially cohort studies with historical controls, should provide compliance data for interventions from both before and after bundle implementation so that the compliance with the bundle in totality can be assessed. The validity of findings from studies that do not present compliance data must be questioned. ${ }^{13,14}$

Having so many elements in a care bundle may present an inherent flaw in its implementation and the ability to achieve compliance. Equally, as the DH HII SSI care bundle is spread over pre, intra and postoperative periods, it involves several clinical teams, which may also limit compliance and effectiveness. It was not possible to break the bundle down into its constituent parts.

This study also highlights the need to provide composite compliance data. The Institute for Healthcare Improvement states that the successful implementation of a bundle is dependent on the consistent and systematic application of all elements in a bundle. ${ }^{1}$ This has been demonstrated in a study on the implementation of a VAP bundle, which achieved a significant reduction in VAP that had not been achieved with earlier ad hoc VAP prevention guidelines. ${ }^{15}$ Compliance with the complete bundle must therefore be reported and SSI rates presented for patients who received the entire bundle.

Recording and reporting of compliance data must be robust. Studies have found conflicting outcomes between hospital audit data and direct observation of compliance. ${ }^{16}$ There is some uncertainty around using data that have been collected for audit purposes, especially if these are directly or indirectly associated with financial outcomes. For example, a study of 300 hospitals in the US found changes in the coding and documentation of SSIs following the decision by Medicare to stop financing the complications arising from SSIs. ${ }^{17}$

Although it was surprising that there was only a minimal increase in compliance, others have also reported a lack of improvement in compliance during care bundle studies. ${ }^{18}$ Similarly, compliance rates with entire SSI care bundles in other studies are also low: $2 \%$ and $21 \% \cdot{ }^{19,20}$ This may be due to insufficient engagement with staff around the development and implementation of the bundle, training issues or the availability of equipment when required. Studies that include multidisciplinary stakeholder groups, steering committees, regular focus groups and newsletter updates have shown higher levels of compliance. ${ }^{21}$

If the success of a SSI care bundle is ultimately dependent on staff participation, then perhaps staff engagement should be listed as a bundle intervention; in this study, we did have senior staff support and gave monthly feedback. This could possibly have been improved further by the use of a steering committee, regular focus groups or newsletter updates. Following a lack of compliance improvement in care bundles to reduce CLABSI, an integrated approach model was developed, which includes engagement and education of all staff and stakeholders. ${ }^{22}$

The only individual intervention in this study that stood out as being effective in reducing SSIs was preoperative warming. Trials reporting the positive effects of preoperative warming first emerged over ten years ago and yet hospitals have been slow to implement local or systemic preoperative warming. ${ }^{23}$ While national SSI care bundles in both the UK and the US include 'maintaining normothermia' as an intervention, neither specifically recommend using routine preoperative warming.

\section{Study limitations}

The duration of the implementation phase of the study was comparatively short (six months), and compliance may have increased had the study continued for a longer period and included more patients. Furthermore, although some of the elements of the DH HII bundle (hair removal, antibiotic prophylaxis and maintenance of normothermia) have a level I evidence base, other elements have varying grades of supporting evidence. 


\section{Conclusions}

Care bundles to reduce SSIs after colorectal surgery have been shown to be effective but this current evaluation of the DH HII care bundle found no reduction in SSIs. Increased staff education and engagement may help with this, in addition to monthly posting of SSI rates and bundle compliance data in key areas. However, compliance with interventions did not increase following the introduction of the bundle. All studies evaluating the effectiveness of care bundles must include compliance data for interventions from both before and after bundle implementation. Further evaluation of the DHI HII bundle must be carried out as it is resource intensive to implement.

\section{Acknowledgements}

The authors would like to thank the SSI surveillance staff of the two hospitals in which the study was undertaken, for the use of their anonymised data. They are also grateful for research grants from CareFusion and 3M, which helped to fund the study.

\section{References}

1. What Is a Bundle? Institute for Healthcare Improvement. http://www.ihi.org/ resources/Pages/ImprovementStories/WhatlsaBundle.aspx (cited January 2016).

2. Resar R, Pronovost P, Haraden C et al. Using a bundle approach to improve ventilator care processes and reduce ventilator-associated pneumonia. Jt Comm $J$ Qual Patient Saf 2005; 31: 243-248.

3. Pronovost $\mathrm{P}$, Needham $\mathrm{D}$, Berenholtz $\mathrm{S}$ et al. An intervention to decrease catheter-related bloodstream infections in the ICU. N Engl J Med 2006; 355 ; 2,725-2,732.

4. Bratzler DW, Hunt DR. The surgical infection prevention and surgical care improvement projects: national initiatives to improve outcomes for patients having surgery. Clin Infect Dis 2006; 43: 322-330.

5. High Impact Interventions. Department of Health. http://hcai.dh.gov.uk/ whatdoido/high-impact-interventions/ (cited January 2016).

6. Weston A, Caldera K, Doron S. Surgical care improvement project in the value-based purchasing era: more harm than good? Clin Infect Dis 2013; 56: 424-427.

7. Berenguer CM, Ochsner MG, Lord SA, Senkowski CK. Improving surgical site infections: using National Surgical Quality Improvement Program data to institute Surgical Care Improvement Project protocols in improving surgical outcome. J Am Coll Surg 2010; 210: 737-741.

8. Hawn MT, Vick CC, Richman J et al. Surgical site infection prevention: time to move beyond the surgical care improvement program. Ann Surg 2011; 254: 494-499.

9. National Institute for Health and Clinical Excellence. Surgical Site Infections: Prevention and Treatment. London: NICE; 2008.

10. Tanner J, Padley W, Assadian $\mathrm{O}$ et al. Do surgical care bundles reduce the risk of surgical site infections in patients undergoing colorectal surgery? A systematic review and cohort meta-analysis of 8,515 patients. Surgery 2015; 158 : 66-77.

11. Health Protection Agency. Protocol for the Surveillance of Surgical Site Infection. London: HPA; 2008.

12. Horan TC, Gaynes RP, Martone WJ et al. CDC definitions of nosocomial surgical site infections, 1992: a modification of CDC definitions of surgical wound infections. Infect Control Hosp Epidemiol 1992; 13: 606-608.

13. Lutfiyya W, Parsons, D, Breen J. A colorectal 'care bundle' to reduce surgical site infections in colorectal surgeries: a single-center experience. Perm J 2012; 16: $10-16$.

14. Rasouli MR, Jaberi MM, Hozack WJ et al. Surgical care improvement project (SCIP): has its mission succeeded? J Arthroplasty 2013; 28: 1,072-1,075.

15. Morris AC, Hay AW, Swann DG et al. Reducing ventilator-associated pneumonia in intensive care: impact of implementing a care bundle. Crit Care Med 2011; 39: 2,218-2,224.

16. Pickering SP, Robertson ER, Griffin D et al. Compliance and use of the World Health Organization checklist in UK operating theatres. Br J Surg 2013; 100: $1,664-1,670$.

17. Hartmann CW, Huff T, Palmer JA et al. The Medicare policy of payment and adjustment for health care-associated infections: perspectives on potential unintended consequences. Med Care Res Rev 2012; 69: 45-61.

18. Larochelle M, Hyman N, Gruppi L, Osler T. Diminishing surgical site infections after colorectal surgery with surgical care improvement project: is it time to move on? Dis Colon Rectum 2011; 54: 394-400.

19. Waits SA, Fritze D, Banerjee M et al. Developing an argument for bundled interventions to reduce surgical site infection in colorectal surgery. Surgery 2014; 155: 602-606

20. Bull A, Wilson J, Worth LJ et al. A bundle of care to reduce colorectal surgical infections: an Australian experience. J Hosp Infect 2011; 78: 297-301.

21. Crolla RM, van der Laan L, Veen EJ et al. Reduction of surgical site infections after implementation of a bundle of care. PLoS One 2012; 7: e44599.

22. Provonost PJ, Berenholtz SM, Needham DM. Translating evidence into practice: a model for large scale knowledge translation. BMJ 2008; 337: a1714.

23. Melling AC, Ali B, Scott EM, Leaper DJ. Effects of preoperative warming on the incidence of wound infection after clean surgery: a randomised controlled trial. Lancet 2001; 358: 876-880 